\title{
Cost-effectiveness analysis of quadrivalent influenza vaccine versus trivalent influenza vaccine for elderly in Hong Kong
}

\author{
Joyce HS You ${ }^{1 *}$, Wai-kit Ming ${ }^{1}$ and Paul KS Chan ${ }^{2,3}$
}

\begin{abstract}
Background: Cost and quality-adjusted life-years (QALYs) gained by quadrivalent influenza vaccine (QIV) versus trivalent influenza vaccine (TIV) in Hong Kong elderly were estimated over 9 seasons.

Methods: TIV-unmatched influenza B infection rates with QIV versus TIV were estimated by an epidemiology model. Model parameters included percentages of influenza B lineages in circulation, influenza B-associated hospital admission, age-specific population, vaccine coverage and effectiveness. Incremental cost per QALY gained (ICER) by QIV versus TIV were estimated from Hong Kong's societal perspective.

Results: Mean reduction in influenza B infection rate was 191.3 (95\%Cl 45.1-337.5) per 100,000 population aged $\geq 65$ years. Highest cost savings and QALYs gained by QIV occurred in 2007 with high percentage of TIV-unmatched strain (92.9\%) for age groups 65-79 years (USD266,473 and 22.8 QALYs) and $\geq 80$ years (USD483,461 and 27.3 QALYs). ICERs of QIV were below willingness-to-pay for age group 65-79 years in 6, 5 and 3 years when QIV cost + USD1 + USD2 and + USD5 more than TIV, respectively. For age group $\geq 80$ years, ICERs of QIV were below willingness-to-pay in 7 and 5 years when QIV cost + USD1 and + USD5, correspondingly.
\end{abstract}

Conclusions: Acceptance of QIV to be cost-effective in Hong Kong elderly was subject to QIV unit cost and percentage of circulating TIV-unmatched influenza B lineages.

Keywords: Quadrivalent influenza vaccine, Trivalent influenza vaccine, Elderly, Hong Kong, Cost-effectiveness analysis

\section{Background}

We recently reported the percentages of influenza $B$ lineages (B/Victoria and $\mathrm{B} /$ Yamagata) in clinical isolates in 2000-2010 and found that co-circulation of both influenza B lineages was very common in Hong Kong [1]. Furthermore, one of the influenza B lineages predominated (accounting for over $80 \%$ of the circulating influenza B strains) in six of the ten years. These predominated years were associated with increased risk of hospitalization by 1.4 -fold. The seasonal trivalent influenza vaccine (TIV) covers two subtypes of influenza A (A/H1N1 and A/H3N2) plus one of the two influenza $B$ lineages circulating in humans, and TIV only matched the predominated

\footnotetext{
* Correspondence: joyceyou@cuhk.edu.hk

${ }^{1}$ School of Pharmacy, Faculty of Medicine, The Chinese University of Hong Kong, Shatin, NT, Hong Kong

Full list of author information is available at the end of the article
}

influenza B strain in two of these six predominated years.

Findings in literature suggested that including two lineages of influenza $B$ in a quadrivalent influenza vaccine (QIV) would have a positive impact in terms of cost saving and reducing hospitalization related to influenza infection $[2,3]$. To further quantify the clinical and economic impact of QIV, we estimated the difference in costs and qualityadjusted life-years (QALYs) gained by QIV, when compared to TIV, in elderly population over the period of 2001-2010 from a societal perspective of Hong Kong.

\section{Methods}

In the present analysis, we estimated the potential cost and QALYs difference of influenza B, when compared to TIV, from 2001-2010 years in elderly population (aged $\geq 65$ years) in Hong Kong. Year 2009 was excluded as it was dominated by the novel pandemic influenza $\mathrm{A}$ 
2009 H1N1. Events caused by infection of the TIVunmatched influenza B lineage in each year were calculated by an epidemiology model. The infections caused by the influenza B lineage or influenza A subtypes covered by both TIV and QIV were excluded, assuming that there were no change in the infection rates. The costs and QALYs loss for symptomatic patients who received medical care in outpatient and hospital setting were estimated from the event rates. The parameters used for estimation of clinical events, cost difference and QALYs gained by QIV versus TIV were listed in Table 1. All model parameters were obtained from published literature.

\section{Clinical events analysis}

For each year, the hospitalization rate of target $B$ lineage was calculated using the influenza $B$ hospital admission rate and the percentage of target influenza B lineage identified in the isolates reported in our previous cohort study [1]. Briefly, in this prior study we identified hospitalized patients with laboratory confirmed influenza virus A or B infection

Table 1 Parameters for calculation of event rates, costs and QALYs with QIV versus TIV

\begin{tabular}{|c|c|c|}
\hline Parameter & Values & References \\
\hline \multicolumn{3}{|l|}{ Clinical inputs } \\
\hline Influenza vaccine coverage ( $\geq 65$ years) & $39.1 \%$ & 15 \\
\hline TIV vaccine effectiveness against influenza B (2001-2010) & $0.45-0.75$ & 7-14 \\
\hline Reduction in TIV influenza B vaccine effectiveness against TIV-unmatched influenza B lineage & $30 \%$ & 6 \\
\hline Influenza B hospitalization rate (2001-2010) by age group (per 100,000): & & 1 \\
\hline $65-79$ years & $1-40.8$ & \\
\hline$\geq 80$ years & $0-222.1$ & \\
\hline Hong Kong population (2001-2010) by age group: & & 24 \\
\hline $65-79$ years & $600,986-665,642$ & \\
\hline$\geq 80$ years & $146,066-257,966$ & \\
\hline Influenza B lineage in circulation (2001-2010) & & 1 \\
\hline Yamagata & $5.3 \%-96.3 \%$ & \\
\hline Victoria & $3.7-94.7 \%$ & \\
\hline Case-fatality ratio & $0.05 \%$ & 5 \\
\hline Percent of inpatient cases with ICU admission: & $2.4 \%$ & 4 \\
\hline Mortality rate of influenza $B$ in $\geq 65$ years inpatient cases & $5.9 \%$ & 1 \\
\hline \multicolumn{3}{|l|}{ Utility } \\
\hline Utility score for $>65$ years & 0.84 & 25 \\
\hline Utility loss & & $16-22$ \\
\hline Outpatient care & 0.40 & \\
\hline Hospitalization without ICU care & 0.50 & \\
\hline Hospitalization with ICU care & 0.62 & \\
\hline Life expectancy in Hong Kong (years) & 83 & 24 \\
\hline \multicolumn{3}{|l|}{ Cost (USD)* } \\
\hline Cost of outpatient clinic (per visit) & 49 & 26 \\
\hline Number of clinic visit for patients without hospitalization & 1 & Assumption \\
\hline Cost of hospitalization without ICU care (per day) & 600 & 26 \\
\hline Cost of hospitalization with ICU care (per day) & 2,949 & 26 \\
\hline \multicolumn{3}{|l|}{ Length of illness (days) } \\
\hline Outpatient care & 7 & 23 \\
\hline Hospitalization (length of stay) & 10.8 & 4 \\
\hline Caregiver salary per day & 47 & 24 \\
\hline Labor force rate & $68 \%$ & 24 \\
\hline Unemployment rate & $3.7 \%$ & 24 \\
\hline
\end{tabular}

*1 USD $=7.8$ HKD. 
and the circulation pattern of influenza B lineage in the Prince of Wales Hospital (a 1300-bed Hong Kong teaching hospital), and calculated age-stratified population-based hospitalization rates of influenza A and B for years from 2000-2010 (excluding 2009 for the same reason above).

The intensive care unit (ICU) admission rate was calculated by hospitalization rate of target lineage together with ICU admission rate among hospitalized patients [4]. The mortality rate of influenza B among hospitalized patients was retrieved from patients aged $\geq 65$ years in the same cohort of our previous study [1]. The expected annual infection rate of target lineage was estimated using the mortality rate and a case-fatality ratio of influenza [5]. The rate of outpatient care was further calculated as the difference of estimated annual infection rate and hospitalization rate. To estimate the reduction in event rates of outpatient care, hospitalization, ICU admission and death with the use of QIV versus TIV, the epidemiology model described by Reed et al. was adoped [2]:

(1) Expected event rate without influenza vaccination $=$ Event rate with $\mathrm{TIV} /\left(1-\left(\mathrm{VC} \times \mathrm{VE}_{\mathrm{TIV}}\right)\right)$

(2) Expected event rate with QIV = expected event rate without influenza vaccination $\times\left(1-\left(\mathrm{VC} \times \mathrm{VE}_{\mathrm{QIV}}\right)\right)$ where $\mathrm{VC}$ and $\mathrm{VE}$ were the vaccine coverage and vaccine effectiveness against the TIV-unmatched lineage, respectively.

(3) Reduction in event rate = Event rate with TIV expected event rate with QIV.

The VE $\mathrm{VIIV}_{\mathrm{T}}$ against the TIV-unmatched influenza $\mathrm{B}$ lineage was estimated to be reduced by $30 \%$ [6]. The $\mathrm{VE}_{\mathrm{QIV}}$ against the TIV-unmatched influenza $\mathrm{B}$ lineage was assumed to be the same as VE of TIV against the TIV-selected influenza B lineage, and the year-specific VE was retrieved from literature reported in the period of 2001-2010 [7-14]. The year 2012 age-specific influenza vaccine coverage rate $(39.1 \%)$ in elderly published by the Department of Health of Hong Kong was applied in the present analysis for 9 seasons [15].

The reduction in case numbers of each event (outpatient care, hospitalization, ICU admission and death) were then calculated by multiplying the change in event rate and the population of $\geq 65$ years for each year.

\section{QALY analysis}

The QALYs gained by QIV were calculated using the number of reduced infected cases, and the utility value and duration of time-spent in each of the four statuses: (1) Outpatient care, (2) hospitalization without ICU admission, (3) ICU care, and (4) death. The utilities were retrieved from health-related quality of life reported from literature (Table 1) [16-22]. The time-spent in outpatient care and hospitalization were the length of outpatient duration of illness [23] and length of hospital stay [4], correspondingly. The QALYs loss of death was estimated using life expectancy in Hong Kong and age-specific utility value for $\geq 65$ years $[24,25]$. The QALYs loss in both vaccine arms was discounted to 2014 with $3 \%$ annual discounted rate.

\section{Cost analysis}

Both direct medical cost and indirect cost of reduced cases of the TIV-unmatched influenza B lineage were considered as cost savings of QIV, from the societal perspective of Hong Kong. Direct medical cost included costs of outpatient care and hospitalization. The cost per outpatient clinic visit and cost per hospital day (with and without ICU care) were estimated from the 2014 charges of the Hospital Authority (HA). HA is the largest, nonprofit-making public health organization in Hong Kong. Assuming the charges listed by the Hospital Authority represent only the cost components (including labor costs) with no addition of profits, the cost per general outpatient visit and daily cost of hospitalization were therefore approximated using the Hospital Authority charges [26].

Productivity loss of caregivers of elderly patients was estimated by the median daily income (non-gender specific) of the population in Hong Kong [24], and duration of hospitalization for inpatient care. For patients receiving only outpatient care, the duration of productivity loss was limited to days attending outpatient clinic. The number of clinic visit was assumed to be one time for a conservative estimation on both direct and indirect costs of outpatient care. The proportion of employed caregivers was estimated by the percentage of Hong Kong population in the labor force and the employment rate among the labor force [24].

\section{Cost-effectiveness analysis}

The incremental cost per QALY gained (ICER) by QIV versus TIV was calculated using the following equation:

(Additional vaccine cost of QIV versus TIV - Costsavings of QIV)/QALYs gained by reduced events, where additional vaccine cost of QIV was calculated by:

Additional unit cost of QIV versus TIV $\times$ age-specific coverage rate $\times$ population of age group

The current unit cost of TIV in Hong Kong is USD7.7. Four levels of additional cost (the unit cost of QIV to be USD1, 2, 5 and 10 more than TIV) were considered in the cost-effectiveness analysis. As recommended by the World Health Organization (WHO), three-fold of gross domestic product (GDP) per capita was used as the threshold of willingness-to-pay per QALY [27]. A scenario with ICER of QIV less than 3-fold of GDP per capita of Hong Kong was considered as cost-effective. 


\section{Results}

The reduction in expected events rates of TIV-unmatched influenza B by QIV versus TIV are shown in Table 2. The mean reduction in influenza infection rate for elderly aged $\geq 65$ years was 191.3 (95\%CI 45.1-337.5) per 100,000 in 2001-2010. Age-specific reduction in the age group 65-79 years was 104.8 (95\%CI 27.2-182.4) per 100,000 , and $451.4(95 \%$ CI $87.7-815.1)$ per 100,000 in population aged $\geq 80$ years.

The highest total cost savings and QALYs gained by QIV in age group 65-79 years occurred in year 2007 (USD266,473 and 22.8 QALYs), followed by year 2004 (USD256,127 and 21.9 QALYs), and year 2008 (USD191,105 and 16.4 QALYs) (Table 3). Similarly, QIV had the highest cost-saving and QALYs gained from reduced cases of influenza $B$ in age group $\geq 80$ years in 2007 (USD483,461 and 27.3 QALYs), year 2008 (USD344,103 and 19.5 QALYs) and year 2004 (USD218,829 and 12.4 QALYs). In the year 2002, no influenza B virus was identified in hospital admissions at the Prince of Wales Hospital for age groups $65-79$ years and $\geq 80$ years. The expected reductions in

Table 2 Reduction in expected event rates of TIV-unmatched influenza B by QIV versus TIV in Hong Kong elderly

\begin{tabular}{lllll}
\hline Year & \multicolumn{2}{c}{ Rate reduction (per 100,000) } & & \\
\cline { 2 - 5 } & Symptomatic influenza & Outpatient & Hospitalization & Dead \\
\hline Age $65-79$ years & & & \\
2001 & 2.6809 & 2.6582 & 0.0227 & 0.0013 \\
$2002^{*}$ & 0.0000 & 0.0000 & 0.0000 & 0.0000 \\
2003 & 3.7933 & 3.7611 & 0.0321 & 0.0019 \\
2004 & 282.0652 & 279.6748 & 2.3904 & 0.1410 \\
2005 & 84.4697 & 83.7538 & 0.7158 & 0.0422 \\
2006 & 27.5809 & 27.3472 & 0.2337 & 0.0138 \\
2007 & 282.9799 & 280.5818 & 2.3981 & 0.1415 \\
2008 & 201.6311 & 199.9223 & 1.7087 & 0.1008 \\
2010 & 58.1889 & 57.6958 & 0.4931 & 0.0291 \\
Age $\geq 80$ years & & & \\
2001 & 1.9449 & 1.9285 & 0.0165 & 0.0010 \\
$2002^{*}$ & 0.0000 & 0.0000 & 0.0000 & 0.0000 \\
2003 & 42.1055 & 41.7487 & 0.3568 & 0.0211 \\
2004 & 838.6402 & 831.5331 & 7.1071 & 0.4193 \\
2005 & 204.2730 & 202.5419 & 1.7311 & 0.1021 \\
2006 & 49.7802 & 49.3583 & 0.4219 & 0.0249 \\
2007 & 1540.4373 & 1527.3828 & 13.0546 & 0.7702 \\
2008 & 1032.5602 & 1023.8097 & 8.7505 & 0.5163 \\
2010 & 352.9492 & 349.9581 & 2.9911 & 0.1765 \\
\hline
\end{tabular}

*In 2002, no influenza B virus was identified from patients admitted to the Prince of Wale Hospital for age groups $65-79$ years and $\geq 80$ years. The expected reductions in event rates were considered as zero.
Table 3 QALY gained and cost savings of QIV versus TIV

\begin{tabular}{lllll}
\hline Year & QALYs & Direct cost & Indirect cost & Total cost \\
\hline Age $65-79$ & years & & & \\
2001 & 0.199 & 1,781 & 543 & 2,324 \\
$2002^{*}$ & 0 & 0 & 0 & 0 \\
2003 & 0.291 & 2,600 & 792 & 3,392 \\
2004 & 21.940 & 196,311 & 59,816 & 256,127 \\
2005 & 6.670 & 59,679 & 18,184 & 77,863 \\
2006 & 2.210 & 19,777 & 6,026 & 25,803 \\
2007 & 22.826 & 204,241 & 62,232 & 266,473 \\
2008 & 16.370 & 146,474 & 44,631 & 191,105 \\
2010 & 4.785 & 42,818 & 13,047 & 55,864 \\
Age $\geq \mathbf{8 0}$ years & & & \\
2001 & 0.023 & 314 & 96 & 410 \\
$2002^{*}$ & 0 & 0 & 0 & 0 \\
2003 & 0.581 & 7,880 & 2,401 & 10,281 \\
2004 & 12.372 & 167,723 & 51,105 & 218,829 \\
2005 & 3.207 & 43,477 & 13,247 & 56,724 \\
2006 & 0.829 & 11,234 & 3,423 & 14,657 \\
2007 & 27.335 & 370,553 & 112,907 & 483,461 \\
2008 & 19.455 & 263,741 & 80,362 & 344,103 \\
2010 & 7.425 & 100,651 & 30,668 & 131,319 \\
\hline
\end{tabular}

*In 2002, no influenza B virus was identified from patients admitted to the Prince of Wale Hospital for age groups $65-79$ years and $\geq 80$ years. The expected reductions in infected cases, cost-saving and additional QALYs gained with QIV were considered as zero.

infection, cost-saving and additional QALYs gained by QIV were considered to be zero in 2002.

The GDP per capita of Hong Kong was USD36,557. ${ }^{32}$ Using 3-time GDP per capita (USD109,671) as the threshold of willingness-to-pay, ICERs of QIV indicated that it was more cost-effective than TIV for age group 65-79 years in 6 years when QIV cost USD1 more than TIV (Table 4). When QIV was more costly than TIV by USD2, USD5 and USD10, it was more cost-effective than TIV in 5, 3 and 2 years, respectively. In the age group $\geq 80$ years, the ICERs of QIV was below the threshold of willingness-to-pay in 7 years when QIV versus TIV cost level was + USD1. QIV remained to be cost-effective in 5 years when it cost up to USD5 more than TIV. It was cost-effective in 3 years when it cost USD10 more than TIV.

\section{Discussion}

The present analysis quantified the expected reduction in TIV-unmatched influenza B infection rate, direct medical cost and indirect cost of productivity loss, and QALYs loss if QIV was available in Hong Kong during 2001-2010. The symptomatic infection rates were reduced by 191.3 (95\%CI 45.1-337.5) per 100,000 elderly 
Table 4 ICER" of QIV versus TIV

\begin{tabular}{|c|c|c|}
\hline \multirow{2}{*}{$\begin{array}{l}\text { Year } \\
\text { Age (years) }\end{array}$} & \multicolumn{2}{|l|}{ ICER } \\
\hline & $65-79$ & $\geq 80$ \\
\hline \multicolumn{3}{|c|}{ Additional cost of $Q I V=U S D \$ 1$} \\
\hline 2001 & $1,168,835$ & $2,447,575$ \\
\hline 2002 & $N E^{*}$ & $N E^{*}$ \\
\hline 2003 & 822,641 & 96,189 \\
\hline 2004 & -454 & $-11,969$ \\
\hline 2005 & 25,793 & 5,786 \\
\hline 2006 & 103,072 & 78,633 \\
\hline 2007 & -490 & $-14,574$ \\
\hline 2008 & 4,022 & $-13,043$ \\
\hline 2010 & 42,714 & $-4,102$ \\
\hline \multicolumn{3}{|c|}{ Additional cost of $Q I V=U S D \$ 2$} \\
\hline 2001 & $2,349,345$ & $4,912,836$ \\
\hline 2002 & $N E^{*}$ & $N E^{*}$ \\
\hline 2003 & $1,656,956$ & 210,065 \\
\hline 2004 & 10,766 & $-6,252$ \\
\hline 2005 & 63,259 & 29,258 \\
\hline 2006 & 217,818 & 174,952 \\
\hline 2007 & 10,694 & $-11,461$ \\
\hline 2008 & 19,718 & $-8,400$ \\
\hline 2010 & 97,103 & 9,483 \\
\hline \multicolumn{3}{|c|}{ Additional cost of $Q I V=U S D \$ 5$} \\
\hline 2001 & $5,890,874$ & $12,308,620$ \\
\hline 2002 & $N E^{*}$ & $N E^{*}$ \\
\hline 2003 & $4,159,902$ & 551,693 \\
\hline 2004 & 44,426 & 10,900 \\
\hline 2005 & 175,659 & 99,676 \\
\hline 2006 & 562,056 & 463,911 \\
\hline 2007 & 44,245 & $-2,124$ \\
\hline 2008 & 66,806 & 5,531 \\
\hline 2010 & 260,268 & 50,238 \\
\hline \multicolumn{3}{|c|}{ Additional cost of QIV $=$ USD $\$ 10$} \\
\hline 2001 & $11,793,422$ & $24,634,926$ \\
\hline 2002 & $N E^{*}$ & $N E^{*}$ \\
\hline 2003 & $8,331,478$ & $1,121,073$ \\
\hline 2004 & 100,527 & 39,487 \\
\hline 2005 & 362,993 & 217,039 \\
\hline 2006 & $1,135,786$ & 945,509 \\
\hline 2007 & 100,164 & 13,440 \\
\hline 2008 & 145,286 & 28,749 \\
\hline 2010 & 532,209 & 118,163 \\
\hline
\end{tabular}

\# ICER = incremental cost per QALY saved by QIV. Using the threshold of 3-time gross domestic product per capita in Hong Kong as the willingness-to-pay per QALY, QIV was cost-effective with ICER USD109,671 or less (bold). A negative value of ICER indicated that QIV was less costly than TIV and gained higher QALYs. ${ }^{*} \mathrm{NE}=$ Not effective. ICER was not calculated in 2002 because the expected reduction in infection and expected QALY gained were zero. aged $\geq 65$ years in the 2001-2010, comparable to the estimated reduction in the US reported by Reed et al. (ranged from 1-440 per 100,000 in 2001-2008) [2].

As demonstrated by the annual cost savings and QALYs loss averted by QIV (Table 2), and QIV would be more cost-effective than TIV if the unit cost of QIV is the same as TIV (additional unit cost= USD0) in all 9 years (except year 2002). The number of years in which QIV was more cost-effective than TIV decreased in both age groups 65-79 years and $\geq 80$ year when the unit cost of QIV increased by USD1-10 (Table 3). Our results were different from the findings of an economic study of QIV in the US [3]. Lee et al. reported positive cost savings (including both direct and indirect costs) by QIV when the unit cost of QIV was USD5-30 more than TIV, based upon infection rate reduction reported by Reed et al. In the present analysis, QIV would cost more to gain QALYs (as indicated by a positive value of ICER) in most of the years when QIV cost more than TIV by USD1-10. The difference was mainly caused by lower healthcare costs in Hong Kong comparing to the US for inpatient (USD1,800-6,600 versus USD4,221-11,372) and outpatient care (USD49 versus USD76-102). For every case of infection averted, less direct cost of treatment was saved in Hong Kong versus US because of the lower cost of care.

In the present study, QIV was cost-effective in year 2007 at four cost levels (+USD1, +USD2, +USD5 and + USD10). The cost savings and QALYs gained by QIV were the highest in this year. It could be explained by the fact that a high percentage of circulating influenza B lineages in Hong Kong were not covered by TIV (92.9\%) and the hospitalization rate for influenza B in the elderly was the highest in 2007 [1]. The cost-effectiveness of the influenza vaccine tends to be enhanced when the expected hospitalization rate without the vaccine is high [28]. The expected QALYs gained and cost savings of QIV were sufficient to overcome the economic impact of additional cost of QIV and it therefore remained costeffective over a broad range of additional vaccine cost.

Our study was limited by using a single source (one teaching hospital) of influenza B hospital admission rates and circulating virus lineages for the calculation of population-based influenza B infection rate in Hong Kong elderly. The infection rate was estimated by the case-fatality ratio, yet this information was not available from Hong Kong. A case-fatality ratio derived from US was used, as the estimated influenza-related mortality rates were generally similar in Hong Kong and the US $[29,30]$. We assumed that patients in the outpatient setting would seek care at medical clinic for one time, as Chinese older people in Hong Kong tend to perceive influenza as a serious illness [31]. Some patients would use home remedies or over-the-counter preparation to 
alleviate influenza symptoms, and the cost-saving of QIV might be overestimated. On the other hand, the QALYs loss of caregivers was not included in the analysis and the QALY saved by QIV might therefore be underestimated. The current cost and QALYs were calculated based upon the vaccine coverage of $39.1 \%$ and herd immunity effect was not included in the analysis. The study findings are likely to change if the vaccine coverage is improved in elderly (and therefore generates herd immunity), probably showing more prominent saving in cost and QALYs with QIV comparing to TIV. Sensitivity analysis was not conducted in the present study. Expected cost and clinical outcomes were calculated for 9 seasons using year-specific key parameters. The change of cost and clinical outcomes was found to be sensitive to the variation of key year-specific parameters (namely the percentage of circulating TIVunmatched influenza B lineage and hospitalization rate). Using year-specific inputs has therefore served the purpose of sensitivity analysis.

\section{Conclusions}

In conclusion, QIV could reduce direct and indirect costs of influenza B infection and gain higher QALYs when compared to TIV in the elderly population of Hong Kong. The acceptance of QIV to be cost-effective in Hong Kong elderly was highly subject to the unit cost of QIV versus TIV and the percentage of circulating TIV-unmatched influenza B lineages.

\section{Competing interests}

Chan PSK has participated in clinical trials supported by F. Hoffmann-La Roche and GlaxoSmithKline, received consultancy fees from F. Hoffmann-La Roche and GlaxoSmithKline, and served the Quadrivalent Influenza Vaccine Asia-Pacific Advisory Board of GlaxoSmithKline. You JHS and Ming WK have no conflict of interest to disclose.

\section{Authors' contributions}

JHSY and PKSC: Study concept and design, and interpretation of data, preparation of manuscript. JHSY and WKM: Acquisition of data and data analysis. All authors read and approved the final manuscript.

\section{Funding}

This study did not receive any funding.

\begin{abstract}
Author details
'School of Pharmacy, Faculty of Medicine, The Chinese University of Hong Kong, Shatin, NT, Hong Kong. ${ }^{2}$ Department of Microbiology, Faculty of Medicine, The Chinese University of Hong Kong, Shatin, NT, Hong Kong. ${ }^{3}$ Stanley Ho Centre for Emerging Infectious Diseases, Faculty of Medicine, The Chinese University of Hong Kong, Shatin, NT, Hong Kong.
\end{abstract}

Received: 9 June 2014 Accepted: 6 November 2014

Published online: 25 November 2014

\section{References}

1. Chan PK, Chan MC, Cheung JL, Lee N, Leung TF, Yeung ACM, Wong MCS, Ngai KLK, Nelson EAS, Hui DSC: Influenza B lineage circulation and hospitalization rates in a subtropical city, Hong Kong, 2000-2010. Clin Infect Dis 2013, 56:677-684.

2. Reed C, Meltzer Ml, Finelli L, Fiore A: Public health impact of including two lineages of influenza $B$ in a quadrivalent seasonal influenza vaccine. Vaccine 2012, 30:1993-1998.
3. Lee BY, Bartsch SM, Willig AM: The economic value of a quadrivalent versus trivalent influenza vaccine. Vaccine 2012, 30:7443-7446.

4. Lee N, Chan PKS, Lui GCY, Wong BCK, Sin WWY, Choi KW, Wong RYK, Lee ELY, Yeung ACM, Ngai KLK, Chan MCW, Lai RWM, Yu AWY, Hui DSC: Complications and outcomes of pandemic 2009 influenza A (H1N1) virus infection in hospitalized adults: how do they differ from those in seasonal influenza? J Infect Dis 2011, 203:1739-1747.

5. Chowell G, Miller MA, Viboud C: Seasonal influenza in the United States, France, and Australia: transmission and prospects for control. Epidemiol Infect 2008, 136:852-864.

6. Centers for Disease Control and Prevention: Updated adjusted estimates of 2012-2013 seasonal influenza vaccine effectiveness in the United States. Assessed on 30 April 2014: http://www.cdc.gov/vaccines/acip/ meetings/downloads/min-archive/min-jun13.pdf

7. Centers for Disease Control and Prevention: Interim within-season estimate of the effectiveness of trivalent inactivated influenza vaccine-Marshfield, Wisconsin, 2007-08 influenza season. MMWR Morb Mortal Wkly Rep 2008, 57:393-398.

8. Belongia EA, Kieke BA, Donahue JG, Greenlee RT, Balish A, Foust A, Lindstrom S, Shay DK, Marshfield Influenza Study Group: Effectiveness of inactivated influenza vaccines varied substantially with antigenic match from the 2004-2005 season to the 2006-2007 season. J Infect Dis 2009, 199:159-167.

9. Coleman LA, Kieke B, Irving S, Shay DK, Vandermause M, Lindstrom S, Belongia EA: Comparison of influenza vaccine effectiveness using different methods of case detection: clinician-ordered rapid antigen tests vs. active surveillance and testing with real-time reverse-transcriptase polymerase chain reaction (rRT-PCR). Vaccine 2011, 29:387-390.

10. Frey S, Vesikari T, Szymczakiewicz-Multanowska A, Lattanzi M, Izu A, Groth N, Holmes S: Clinical efficacy of cell culture-derived and egg-derived inactivated subunit influenza vaccines in healthy adults. Clin Infect Dis 2010, 51:997-1004.

11. Monto AS, Ohmit SE, Petrie JG, Johnson E, Truscon R, Teich E, Rotthoff J, Boulton M, Victor JC: Comparative efficacy of inactivated and live attenuated influenza vaccines. N Engl J Med 2009, 361:1260-1267.

12. Ohmit SE, Victor JC, Teich ER, Truscon RK, Rotthoff JR, Newton DW, Campbell SA, Boulton ML, Monto AS: Prevention of symptomatic seasonal influenza in 2005-2006 by inactivated and live attenuated vaccines. $\mathrm{J}$ Infect Dis 2008, 198:312-317.

13. Talbot HK, Griffin MR, Chen Q, Zhu Y, Williams JV, Edwards KM: Effectiveness of seasonal vaccine in preventing confirmed influenzaassociated hospitalizations in community dwelling older adults. $J$ Infect Dis 2011, 203:500-508.

14. Treanor JJ, Talbot HK, Ohmit SE, Coleman LA, Thompson MG, Cheng PY, Petrie JG, Lofthus G, Meece JK, Williams JV, Berman L, Breese Hall C, Monto AS, Griffin MR, Belongia E, Shay DK, US Flu-VE Network: Effectiveness of seasonal influenza vaccines in the United States during a season with circulation of all three vaccine strains. Clin Infect Dis 2012, 55:951-959.

15. Chan D: Seasonal influenza vaccination coverage survey for the 2012/ 2013 season. Communicable Diseases Watch 2013, 10:74-77.

16. Smith KJ, Roberts MS: Cost-effectiveness of newer treatment strategies for influenza. Am J Med 2002, 113:300-307.

17. Sander B, Hayden FG, Gyldmark M, Garrison LPJ: Post-exposure influenza prophylaxis with oseltamivir: cost effectiveness and cost utility in families in the UK. Pharmacoeconomics 2006, 24:373-386.

18. Talbird SE, Brogan AJ, Winiarski AP, Sander B: Cost-effectiveness of treating influenza-like illness with oseltamivir in the United States. Am J Health-Syst Pharm 2009, 66:469-480.

19. Mauskopf JA, Cates SC, Griffin AD, Neighbors DM, Lamb SC, Rutherford C: Cost effectiveness of zanamivir for the treatment of influenza in a high risk population in Australia. Pharmacoeconomics 2000, 17:611-620.

20. Tengs TO, Wallace A: One thousand health-related quality-of-life estimates. Med Care 2000, 38:583-637.

21. Sackett $D L$, Torrance $G W$ : The utility of different health states as perceived by the general public. J Chronic Dis 1978, 31:697-704.

22. Rothberg MB, He S, Rose DN: Management of influenza symptoms in healthy adults. J Gen Intern Med 2003, 18:808-815

23. Petrie JG, Ohmit SE, Hohnson E, Cross RT, Monto AS: Efficacy studies of influenza vaccines: Effect of end points used and characteristics of vaccine failures. J Infect Dis 2011, 203:1309-1315.

24. Census and Statistics Department, The Government of Hong Kong SAR. Assessed on 24 October 2013: www.censtatd.gov.hk/ 
25. Gold MR, Franks P, McCoy KI, Fryback DG: Toward consistency in cost-utility analyses: using national measures to create condition-specific values. Med Care 1998, 36:778-792.

26. Fees and Charges, Hospital Authority, Hong Kong. Assessed on 7 October 2013: www.ha.org.hk

27. Cost-effectiveness thresholds. World Health Organization. Assessed on 3 December 2013: www.who.int/choice/costs/CER_thresholds/en/index.html

28. You JHS, Wong WCW, Ip M, Lee NLS, Ho SC: Cost-effectiveness analysis of influenza and pneumococcal vaccination for Hong Kong elderly in long-term care facilities. J Epidemiol Community Health 2009, 63:906-911.

29. Wu P, Goldstein E, Ho LM, Yang L, Nishiura H, Wu JT, Ip DK, Chuang SK, Tsang T, Cowling BJ: Excess mortality associated with influenza A and B virus in Hong Kong, 1998-2009. J Infect Dis 2012, 206:1862-1871.

30. Goldstein E, Vibound C, Charu V, Lipsitch M: Improving the estimation of influenza-related mortality over a seasonal baseline. Epidemiology 2012, 23:829-838.

31. Kwong EW, Lam IO: Chinese older people in Hong Kong: health beliefs about influenza vaccination. Nursing Older People 2008, 20:29-33.

doi:10.1186/s12879-014-0618-9

Cite this article as: You et al:: Cost-effectiveness analysis of quadrivalent influenza vaccine versus trivalent influenza vaccine for elderly in Hong Kong. BMC Infectious Diseases 2014 14:618.

\section{Submit your next manuscript to BioMed Central and take full advantage of:}

- Convenient online submission

- Thorough peer review

- No space constraints or color figure charges

- Immediate publication on acceptance

- Inclusion in PubMed, CAS, Scopus and Google Scholar

- Research which is freely available for redistribution 